\title{
Common Fixed Points for a Countable Family of Set-Valued Mappings with Quasi-Contractive Conditions on Metrically Convex Spaces
}

\author{
Yuexi Jin, Ailian Jin, Yongjie Piao* \\ Department of Mathematics, College of Science, Yanbian University, Yanji, China \\ Email: yuexi1004@163.com, aljin@ybu.edu.cn,*pyj6216@hotmail.com
}

Received December 10, 2013; revised January 10, 2014; accepted January 17, 2014

Copyright (C) 2014 Yuexi Jin, et al. This is an open access article distributed under the Creative Commons Attribution License, which per- mits unrestricted use, distribution, and reproduction in any medium, provided the original work is properly cited. In accordance of the Creative Commons Attribution License all Copyrights (C) 2014 are reserved for SCIRP and the owner of the intellectual property Yuexi Jin, et al. All Copyright (C) 2014 are guarded by law and by SCIRP as a guardian.

\section{ABSTRACT}

In this paper, we consider a countable family of set-valued mappings satisfying some quasi-contractive conditions. We also construct a sequence by the quasi-contractive conditions of mappings and the boundary condition of a closed subset of a metrically convex space, and then prove that the unique limit of the sequence is the unique common fixed point of the mappings. Finally, we give more generalized common fixed point theorems for a countable family of single-valued mappings. The main results generalize and improve many common fixed point theorems for a finite or countable family of single valued or set-valued mappings with quasi-contractive conditions.

\section{KEYWORDS}

\section{Common Fixed Point; Quasi-Contractive; Metrically Convex; Complete}

\section{Introduction}

There have appeared many fixed point theorems for a single-valued self map of a closed subset of a Banach space. However, in many applications, the mapping under considerations is not a self-mapping on a closed subset. In 1976, Assad [1] gave sufficient condition for such single valued mapping to obtain a fixed point by proving a fixed point theorem for Kannan mappings on a Banach space and putting certain boundary conditions on the mapping. Similar results for multi-valued mappings were respectively given by Assad [2] and Assad and Kirk [3]. On the other hand, many authors discussed common fixed point problems [4-7] for finite single or multi-valued mappings on a complete 2-metric convex space or a complete cone metric space respectively. And some authors also discussed common fixed point problems [8-13] for a countable family of self-single-valued mappings with contractive or quasi-contractive conditions on a metric space or a metrically convex space respectively. These results improved and generalized many previous works.

In this paper, we will discuss the existent problems of common fixed points for a countable family of surjective set-valued mappings, which satisfy certain quasi-contractive condition, defined on a complete metrically convex space and obtain some important theorems. The main results in this paper further generalize and improve many common fixed point theorems for single valued or multi-valued mappings with quasi-contractive type conditions.

Through this paper, $(X, d)$ (or $X)$ is a metric space. Let $b c(X)$ denote the families of all bounded closed subset of $X$.

Let $d(x, A)=\inf _{y \in A} d(x, y)$, the distance between $x$ and $A \in b c(X)$.

${ }^{*}$ Corresponding author. 
Definition 1.1. ([8-10]) A metric space $(X, d)$ is said to be metrically convex, if any $x, y \in X$ with $x \neq y$, there exists $z \in X$ such that $z \neq x, \quad z \neq y$ and $d(x, z)+d(z, y)=d(x, y)$.

Lemma 1.1. ([3,8]) If $K$ is a nonempty closed subset of a complete metrically convex space $(X, d)$, then for any $x \in K$ and $y \notin K$, there exists $z \in \partial K$ which satisfies $d(x, z)+d(z, y)=d(x, y)$.

Lemma 1.2. ([13]) If $X$ is a complete metric space and $A \in b c(X)$, then $d(\cdot, A)$ is continuous on $X$. Moreover, we have :

1) $A=\{x \in X: d(x, A)=0\}$;

2) $A \subset B$ if and only if $x \in X, d(x, B) \leq d(x, A)$;

3) for any $x, y \in X, d(x, A) \leq d(x, y)+d(y, A)$.

\section{Main Results}

Theorem 2.1. Let $K$ be a nonempty closed subset of a complete metrically convex space $(X, d)$ with $\operatorname{int} K \neq \varnothing,\left\{T_{i}: X \rightarrow b c(X)\right\}_{i \in \mathbb{N}}$ a countable family of surjective set-valued mappings with nonempty values such that for any $i, j \in \mathbb{N}$ with $i<j$, any $x, y \in X$,

$$
d(x, y) \leq \lambda u_{i, j}(x, y),
$$

where $u_{i, j}(x, y) \in\left\{\frac{d\left(x, T_{i} x\right)+d\left(y, T_{j} y\right)}{2}, \frac{d\left(x, T_{j} y\right)+d\left(y, T_{i} x\right)}{2}\right\}$ and $\lambda \in\left(0, \frac{-3+\sqrt{17}}{2}\right)$ is a constant number.

Furthermore, if $T_{i}\left(K^{C}\right) \cap \partial K=\varnothing$ for all $i \in \mathbb{N}$, and for each $i \in \mathbb{N}$ and $p \in K$ and any $z \in T_{i} p$, there exists $j \in \mathbb{N}$ such that $p \in T_{j} z$, then $\left\{T_{i}\right\}_{i \in \mathbb{N}}$ has a unique common fixed point in $K$.

Proof Take $x_{0} \in K$. We will construct two sequences $\left\{x_{n}\right\}$ and $\left\{x_{n}^{\prime}\right\}$ in the following manner. Since $T_{1}$ is on-to, there exists $x_{1}^{\prime} \in X$ such that $x_{0} \in T_{1} x_{1}^{\prime}$. If $x_{1}^{\prime} \in K$, then put $x_{1}=x_{1}^{\prime}$; if $x_{1}^{\prime} \notin K$, then by Lemma 1.1 there exits $x_{1} \in \partial K$ such that $d\left(x_{0}, x_{1}\right)+d\left(x_{1}, x_{1}^{\prime}\right)=d\left(x_{0}, x_{1}^{\prime}\right)$. For $x_{1}$, since $T_{2}$ is on-to, there exists $x_{2}^{\prime} \in X$ such that $x_{1} \in T_{2} x_{2}^{\prime}$. If $x_{2}^{\prime} \in K$, then put $x_{2}=x_{2}^{\prime}$; if $x_{2}^{\prime} \notin K$, then by Lemma 1.1 there exists $x_{2} \in \partial K$ such that $d\left(x_{1}, x_{2}\right)+d\left(x_{2}, x_{2}^{\prime}\right)=d\left(x_{1}, x_{2}^{\prime}\right)$. Continuing this way, we obtain $\left\{x_{n}\right\}$ and $\left\{x_{n}^{\prime}\right\}$ :

1) $x_{n-1} \in T_{n} x_{n}^{\prime}$;

2) if $x_{n}^{\prime} \in K$, then put $x_{n}=x_{n}^{\prime}$;

3) if $x_{n}^{\prime} \notin K$, then by Lemma 1.1 there exists $x_{n} \in \partial K$ such that $d\left(x_{n-1}, x_{n}\right)+d\left(x_{n}, x_{n}^{\prime}\right)=d\left(x_{n-1}, x_{n}^{\prime}\right)$;

4) $x_{n} \in K$ for all $n=0,1,2, \cdots$.

Let $P=\left\{x_{i} \in\left\{x_{n}\right\}: x_{i}=x_{i}^{\prime}\right\}$ and $Q=\left\{x_{i} \in\left\{x_{n}\right\}: x_{i} \neq x_{i}^{\prime}\right\}$. If there exists $n \in \mathbb{N}$ such that $x_{n} \in Q$, then $x_{n-1}, x_{n+1} \in P$. In fact, By 3) and the definition of $Q$, we have that $x_{n} \neq x_{n}^{\prime}, x_{n} \in \partial K, x_{n}^{\prime} \notin K$. If $x_{n-1} \in Q$, then $x_{n-1} \in \partial K$. On the other hand, since $T_{n}\left(K^{C}\right) \cap \partial K=\varnothing$ and $x_{n-1} \in T_{n} x_{n}^{\prime} \subset T_{n}\left(K^{C}\right)$, hence $x_{n-1} \notin \partial K$, which is a contradiction. If $x_{n+1} \in Q$, then $x_{n+1} \in \partial K$ and $x_{n+1}^{\prime} \notin K$, hence $x_{n} \in T_{n+1} x_{n+1}^{\prime} \subset T_{n+1}\left(K^{C}\right)$, so $x_{n} \notin \partial K$, which is another contradiction.

By the definitions and properties of $P$ and $Q$, we can estimate $d\left(x_{n}, x_{n+1}\right)$ into three cases:

Case I. $x_{n}, x_{n+1} \in P$. In this case, $x_{n}=x_{n}^{\prime}, x_{n+1}=x_{n+1}^{\prime}, x_{n-1} \in T_{n} x_{n}^{\prime}$ and $x_{n} \in T_{n+1} x_{n+1}^{\prime}$. And we have

$$
d\left(x_{n}, x_{n+1}\right)=d\left(x_{n}^{\prime}, x_{n+1}^{\prime}\right) \leq \lambda u_{n, n+1}\left(x_{n}^{\prime}, x_{n+1}^{\prime}\right),
$$

where

$$
\begin{aligned}
& u_{n, n+1}\left(x_{n}^{\prime}, x_{n+1}^{\prime}\right) \in\left\{\frac{d\left(x_{n}^{\prime}, T_{n} x_{n}^{\prime}\right)+d\left(x_{n+1}^{\prime}, T_{n+1} x_{n+1}^{\prime}\right)}{2}, \frac{d\left(x_{n}^{\prime}, T_{n+1} x_{n+1}^{\prime}\right)+d\left(x_{n+1}^{\prime}, T_{n} x_{n}^{\prime}\right)}{2}\right\} . \\
& \text { If } \begin{aligned}
u_{n, n+1}\left(x_{n}^{\prime}, x_{n+1}^{\prime}\right)=\frac{d\left(x_{n}^{\prime}, T_{n} x_{n}^{\prime}\right)+d\left(x_{n+1}^{\prime}, T_{n+1} x_{n+1}^{\prime}\right)}{2}, \text { then } \\
d\left(x_{n}, x_{n+1}\right)=d\left(x_{n}^{\prime}, x_{n+1}^{\prime}\right) \leq \frac{\lambda}{2}\left[d\left(x_{n}^{\prime}, T_{n} x_{n^{\prime}}\right)+d\left(x_{n+1}^{\prime}, T_{n+1} x_{n+1}^{\prime}\right)\right] \\
\leq \frac{\lambda}{2}\left[d\left(x_{n}, x_{n-1}\right)+d\left(x_{n+1}, x_{n}\right)\right] \\
\leq \frac{\lambda}{2} d\left(x_{n}, x_{n-1}\right)+\frac{1}{2} d\left(x_{n+1}, x_{n}\right),
\end{aligned}
\end{aligned}
$$


hence

$$
\begin{aligned}
& d\left(x_{n}, x_{n+1}\right)=d\left(x_{n}^{\prime}, x_{n+1}^{\prime}\right) \leq \lambda d\left(x_{n-1}, x_{n}\right) ; \\
& \text { If } u_{n, n+1}\left(x_{n}^{\prime}, x_{n+1}^{\prime}\right)=\frac{d\left(x_{n}^{\prime}, T_{n+1} x_{n+1}^{\prime}\right)+d\left(x_{n+1}^{\prime}, T_{n} x_{n}^{\prime}\right)}{2} \text {, then } \\
& d\left(x_{n}, x_{n+1}\right)=d\left(x_{n}^{\prime}, x_{n+1}^{\prime}\right) \leq \frac{\lambda}{2}\left[d\left(x_{n}^{\prime}, T_{n+1} x_{n+1}^{\prime}\right)+d\left(x_{n+1}^{\prime}, T_{n} x_{n}^{\prime}\right)\right] \\
& \leq \frac{\lambda}{2}\left[d\left(x_{n}, x_{n}\right)+d\left(x_{n+1}, x_{n-1}\right)\right] \leq \frac{\lambda}{2}\left[d\left(x_{n-1}, x_{n}\right)+d\left(x_{n}, x_{n+1}\right)\right] \\
& \leq \frac{\lambda}{2}\left[d\left(x_{n-1}, x_{n}\right)+d\left(x_{n}, x_{n+1}\right)\right] \text {, }
\end{aligned}
$$

hence

$$
d\left(x_{n}, x_{n+1}\right)=d\left(x_{n}^{\prime}, x_{n+1}^{\prime}\right) \leq \lambda d\left(x_{n-1}, x_{n}\right) .
$$

Therefore, in any situation, we have

$$
d\left(x_{n}, x_{n+1}\right) \leq \lambda d\left(x_{n-1}, x_{n}\right) \text { for all } n \in \mathbb{N} .
$$

Case II. $x_{n} \in P$ and $x_{n+1} \in Q$. In this case, $x_{n}=x_{n}^{\prime}, x_{n+1} \neq x_{n+1}^{\prime}$ and $x_{n-1} \in T_{n} x_{n}^{\prime}$ and $x_{n} \in T_{n+1} x_{n+1}^{\prime}$. And we have

$$
d\left(x_{n}, x_{n+1}^{\prime}\right)=d\left(x_{n}^{\prime}, x_{n+1}^{\prime}\right) \leq \lambda u_{n, n+1}\left(x_{n}^{\prime}, x_{n+1}^{\prime}\right),
$$

where

$$
u_{n, n+1}\left(x_{n}^{\prime}, x_{n+1}^{\prime}\right) \in\left\{\frac{d\left(x_{n}^{\prime}, T_{n} x_{n^{\prime}}\right)+d\left(x_{n+1}^{\prime}, T_{n+1} x_{n+1}^{\prime}\right)}{2}, \frac{d\left(x_{n}^{\prime}, T_{n+1} x_{n+1}^{\prime}\right)+d\left(x_{n+1}^{\prime}, T_{n} x_{n}^{\prime}\right)}{2}\right\}
$$

If $u_{n, n+1}\left(x_{n}^{\prime}, x_{n+1}^{\prime}\right)=\frac{d\left(x_{n}^{\prime}, T_{n} x_{n}^{\prime}\right)+d\left(x_{n+1}^{\prime}, T_{n+1} x_{n+1}^{\prime}\right)}{2}$, then

$$
\begin{aligned}
d\left(x_{n}, x_{n+1}^{\prime}\right) & =d\left(x_{n}^{\prime}, x_{n+1}^{\prime}\right) \leq \frac{\lambda}{2}\left[d\left(x_{n}^{\prime}, T_{n} x_{n}^{\prime}\right)+d\left(x_{n+1}^{\prime}, T_{n+1} x_{n+1}^{\prime}\right)\right] \\
& \leq \frac{\lambda}{2}\left[d\left(x_{n}, x_{n-1}\right)+d\left(x_{n+1}^{\prime}, x_{n}\right)\right] \\
& \leq \frac{\lambda}{2} d\left(x_{n}, x_{n-1}\right)+\frac{1}{2} d\left(x_{n+1}^{\prime}, x_{n}\right),
\end{aligned}
$$

hence

$$
\text { If } \begin{aligned}
& u_{n, n+1}\left(x_{n}^{\prime}, x_{n+1}^{\prime}\right)=\frac{d\left(x_{n}^{\prime}, x_{n+1} x_{n+1}^{\prime}\right)+d\left(x_{n+1}^{\prime}, T_{n} x_{n}^{\prime}\right)}{2} \text {, then } \\
& d\left(x_{n}, x_{n+1}^{\prime}\right)= d\left(x_{n}^{\prime}, x_{n+1}^{\prime}\right) \leq \frac{\lambda}{2}\left[d\left(x_{n}^{\prime}, T_{n+1} x_{n+1}^{\prime}\right)+d\left(x_{n+1}^{\prime}, T_{n} x_{n}^{\prime}\right)\right] \\
& \leq \frac{\lambda}{2}\left[d\left(x_{n}, x_{n}\right)+d\left(x_{n+1}^{\prime}, x_{n-1}\right)\right] \leq \frac{\lambda}{2}\left[d\left(x_{n-1}, x_{n}\right)+d\left(x_{n}, x_{n+1}^{\prime}\right)\right] \\
& \leq \frac{\lambda}{2} d\left(x_{n}, x_{n-1}\right)+\frac{1}{2} d\left(x_{n+1}^{\prime}, x_{n}\right),
\end{aligned}
$$

hence

$$
d\left(x_{n}, x_{n+1}^{\prime}\right)=d\left(x_{n}^{\prime}, x_{n+1}^{\prime}\right) \leq \lambda d\left(x_{n-1}, x_{n}\right) .
$$

Therefore, in any situation, we have 


$$
d\left(x_{n}, x_{n+1}^{\prime}\right) \leq \lambda d\left(x_{n-1}, x_{n}\right) \text { for all } n \in \mathbb{N} .
$$

But $d\left(x_{n}, x_{n+1}\right)+d\left(x_{n+1}, x_{n+1}^{\prime}\right)=d\left(x_{n}, x_{n+1}^{\prime}\right)$, hence we obtain

$$
d\left(x_{n}, x_{n+1}\right) \leq d\left(x_{n}, x_{n+1}^{\prime}\right) \leq \lambda d\left(x_{n-1}, x_{n}\right) \text { for all } n \in \mathbb{N} .
$$

Case III. $x_{n} \in Q$ and $x_{n+1} \in P$. In this case, $x_{n-1} \in P$ by the property of $P$ and $Q$, and $x_{n} \neq x_{n}^{\prime}$, $x_{n+1}=x_{n+1}^{\prime}, \quad x_{n-1} \in T_{n} x_{n}^{\prime}$ and $x_{n} \in T_{n+1} x_{n+1}^{\prime}$. And we have

$$
d\left(x_{n}^{\prime}, x_{n+1}\right)=d\left(x_{n}^{\prime}, x_{n+1}^{\prime}\right) \leq \lambda u_{n, n+1}\left(x_{n}^{\prime}, x_{n+1}^{\prime}\right),
$$

where

$$
u_{n, n+1}\left(x_{n}^{\prime}, x_{n+1}^{\prime}\right) \in\left\{\frac{d\left(x_{n}^{\prime}, T_{n} x_{n}^{\prime}\right)+d\left(x_{n+1}^{\prime}, T_{n+1} x_{n+1}^{\prime}\right)}{2}, \frac{d\left(x_{n}^{\prime}, T_{n+1} x_{n+1}^{\prime}\right)+d\left(x_{n+1}^{\prime}, T_{n} x_{n}^{\prime}\right)}{2}\right\} .
$$

Here, we give two basic properties:

1) since $x_{n} \in Q$, so $d\left(x_{n-1}, x_{n}\right)+d\left(x_{n}, x_{n}^{\prime}\right)=d\left(x_{n-1}, x_{n}^{\prime}\right)$, and hence $d\left(x_{n-1}, x_{n}\right) \leq d\left(x_{n-1}, x_{n}^{\prime}\right)$;

2) since

$$
d\left(x_{n}, x_{n+1}\right) \leq d\left(x_{n}, x_{n}^{\prime}\right)+d\left(x_{n}^{\prime}, x_{n+1}\right) \leq d\left(x_{n-1}, x_{n}\right)+d\left(x_{n}, x_{n}^{\prime}\right)+d\left(x_{n}^{\prime}, x_{n+1}\right)=d\left(x_{n-1}, x_{n}^{\prime}\right)+d\left(x_{n}^{\prime}, x_{n+1}\right),
$$

hence $d\left(x_{n}, x_{n+1}\right)-d\left(x_{n-1}, x_{n}^{\prime}\right) \leq d\left(x_{n}^{\prime}, x_{n+1}\right)$.

If $u_{n, n+1}\left(x_{n}^{\prime}, x_{n+1}^{\prime}\right)=\frac{d\left(x_{n}^{\prime}, T_{n} x_{n}^{\prime}\right)+d\left(x_{n+1}^{\prime}, T_{n+1} x_{n+1}^{\prime}\right)}{2}$, then

$$
\begin{aligned}
d\left(x_{n}^{\prime}, x_{n+1}\right) & =d\left(x_{n}^{\prime}, x_{n+1}^{\prime}\right) \leq \frac{\lambda}{2}\left[d\left(x_{n}^{\prime}, T_{n} x_{n}^{\prime}\right)+d\left(x_{n+1}^{\prime}, T_{n+1} x_{n+1}^{\prime}\right)\right] \\
& \leq \frac{\lambda}{2}\left[d\left(x_{n}^{\prime}, x_{n-1}\right)+d\left(x_{n+1}, x_{n}\right)\right],
\end{aligned}
$$

hence by 2),

$$
d\left(x_{n}, x_{n+1}\right)-d\left(x_{n-1}, x_{n}^{\prime}\right) \leq \frac{\lambda}{2}\left[d\left(x_{n}^{\prime}, x_{n-1}\right)+d\left(x_{n+1}, x_{n}\right)\right]
$$

So by Case II, we obtain

$$
d\left(x_{n}, x_{n+1}\right) \leq \frac{2+\lambda}{2-\lambda} d\left(x_{n-1}, x_{n}^{\prime}\right) \leq \frac{\lambda(2+\lambda)}{2-\lambda} d\left(x_{n-2}, x_{n-1}\right) \text { for all } n \in \mathbb{N}, n \geq 2 ;
$$

If $u_{n, n+1}\left(x_{n}^{\prime}, x_{n+1}^{\prime}\right)=\frac{d\left(x_{n}^{\prime}, T_{n+1} x_{n+1}^{\prime}\right)+d\left(x_{n+1}^{\prime}, T_{n} x_{n}^{\prime}\right)}{2}$, then

$$
\begin{aligned}
d\left(x_{n}^{\prime}, x_{n+1}\right) & =d\left(x_{n}^{\prime}, x_{n+1}^{\prime}\right) \leq \frac{\lambda}{2}\left[d\left(x_{n}^{\prime}, T_{n+1} x_{n+1}^{\prime}\right)+d\left(x_{n+1}^{\prime}, T_{n} x_{n}^{\prime}\right)\right] \\
& \leq \frac{\lambda}{2}\left[d\left(x_{n}^{\prime}, x_{n}\right)+d\left(x_{n+1}, x_{n-1}\right)\right] \leq \frac{\lambda}{2}\left[d\left(x_{n}^{\prime}, x_{n}\right)+d\left(x_{n-1}, x_{n}\right)+d\left(x_{n}, x_{n+1}\right)\right] \\
& \leq \frac{\lambda}{2}\left[d\left(x_{n-1}, x_{n}^{\prime}\right)+d\left(x_{n}, x_{n+1}\right)\right],
\end{aligned}
$$

hence by 2),

$$
d\left(x_{n}, x_{n+1}\right)-d\left(x_{n-1}, x_{n}^{\prime}\right) \leq \frac{\lambda}{2}\left[d\left(x_{n}^{\prime}, x_{n-1}\right)+d\left(x_{n+1}, x_{n}\right)\right]
$$

So by Case II again, we obtain

$$
d\left(x_{n}, x_{n+1}\right) \leq \frac{2+\lambda}{2-\lambda} d\left(x_{n-1}, x_{n}^{\prime}\right) \leq \frac{\lambda(2+\lambda)}{2-\lambda} d\left(x_{n-2}, x_{n-1}\right) \text { for all } n \in \mathbb{N}, n \geq 2 .
$$

Hence in any situation, we have 


$$
d\left(x_{n}, x_{n+1}\right) \leq \frac{\lambda(2+\lambda)}{2-\lambda} d\left(x_{n-2}, x_{n-1}\right) \text { for all } n \in \mathbb{N}, n \geq 2 .
$$

Therefore, from Case I, Case II and Case III, we obtain

$$
d\left(x_{n}, x_{n+1}\right) \leq \frac{\lambda(2+\lambda)}{2-\lambda} \max \left\{d\left(x_{n-1}, x_{n}\right) d\left(x_{n-2}, x_{n-1}\right)\right\} \text { for all } n \in \mathbb{N}, n \geq 2 .
$$

Let $h=\frac{\lambda(2+\lambda)}{2-\lambda}$, then $0<h<1$ since $\lambda \in\left(0, \frac{-3+\sqrt{17}}{2}\right)$, hence we have

$$
d\left(x_{n}, x_{n+1}\right) \leq h \max \left\{d\left(x_{n-1}, x_{n}\right) d\left(x_{n-2}, x_{n-1}\right)\right\} \text { for all } n \in \mathbb{N}, n \geq 2,
$$

so

$$
d\left(x_{n}, x_{n+1}\right) \leq h^{\frac{n}{2}-1} \max \left\{d\left(x_{2}, x_{1}\right) d\left(x_{1}, x_{0}\right)\right\} \text { for all } n \in \mathbb{N}, n \geq 2 .
$$

Let $\delta=h^{-1} \max \left\{d\left(x_{2}, x_{1}\right), d\left(x_{1}, x_{0}\right)\right\}$, then for $m>n \geq N \geq 2$, $d\left(x_{n}, x_{m}\right) \leq \sum_{i=n}^{m-1} d\left(x_{i}, x_{i+1}\right) \leq \sum_{i=N}^{\infty} d\left(x_{i}, x_{i+1}\right) \leq \sum_{i=N}^{\infty} h^{\frac{i}{2}} \delta \rightarrow 0 \quad$ as $N \rightarrow \infty$. Hence $\left\{x_{n}\right\}_{n \in \mathbb{N}}$ is a Cauchy sequence. Since $X$ is complete, $\left\{x_{n}\right\}$ has a limit $x^{*}$. But $K$ is closed and $x_{n} \in K$ for all $n \in \mathbb{N}$, hence $x^{*} \in K$.

By the property of $P$ and $Q$, we can see that there exists an infinite subsequence $\left\{x_{n_{k}+1}\right\}$ of $\left\{x_{n}\right\}$ such that $x_{n_{k}+1} \in P$, hence $x_{n_{k}+1}=x_{n_{k}+1}^{\prime}$ and $x_{n_{k}} \in T_{n_{k}+1} x_{n_{k}+1}^{\prime}$.

Next, we will prove that $x^{*}$ is a common fixed point of $\left\{T_{i}\right\}_{i \in \mathbb{N}}$. Fix any $i \in \mathbb{N}$, for each fixed $z \in T_{i} x^{*}$, there exists $j \in \mathbb{N}$ such that $x^{*} \in T_{j} z$. Take an enough large $k \in \mathbb{N}$ such that $n_{k}+1>i$ and $n_{k}+1>j$. By Lemma 1.23 ) and (1), we have

$$
d\left(T_{i} x^{*}, x^{*}\right) \leq d\left(T_{i} x^{*}, x_{n_{k}+1}\right)+d\left(x_{n_{k}+1}, x^{*}\right) \leq d\left(z, x_{n_{k}+1}\right)+d\left(x_{n_{k}+1}, x^{*}\right),
$$

and

$$
d\left(z, x_{n_{k}+1}\right) \leq \lambda u_{j, n_{k}+1}\left(z, x_{n_{k}+1}\right),
$$

where

$$
u_{j, n_{k}+1}\left(z, x_{n_{k}+1}\right) \in\left\{\frac{d\left(z, T_{j} z\right)+d\left(x_{n_{k}+1}, T_{n_{k}+1} x_{n_{k}+1}\right)}{2}, \frac{d\left(z, T_{n_{k}+1} x_{n_{k}+1}\right)+d\left(x_{n_{k}+1}, T_{j} z\right)}{2}\right\} .
$$

If $u_{j, n_{k}+1}\left(z, x_{n_{k}+1}\right)=\frac{d\left(z, T_{j} z\right)+d\left(x_{n_{k}+1}, T_{n_{k}+1} x_{n_{k}+1}\right)}{2}$, then

$$
d\left(T_{i} x^{*}, x^{*}\right) \leq \frac{\lambda}{2}\left[d\left(z, T_{j} z\right)+d\left(x_{n_{k}+1}, T_{n_{k}+1} x_{n_{k}+1}\right)\right]+d\left(x_{n_{k}+1}, x^{*}\right) \leq \frac{\lambda}{2}\left[d\left(z, x^{*}\right)+d\left(x_{n_{k}+1}, x_{n_{k}}\right)\right]+d\left(x_{n_{k}+1}, x^{*}\right) .
$$

Let $k \rightarrow \infty$, then $d\left(T_{i} x^{*}, x^{*}\right) \leq \frac{\lambda}{2} d\left(z, x^{*}\right)$ since $x_{n} \rightarrow x^{*}$, hence $d\left(T_{i} x^{*}, x^{*}\right) \leq \frac{\lambda}{2} \inf _{z \in T_{i} x^{*}} d\left(z, x^{*}\right)=\frac{\lambda}{2} d\left(T_{i} x^{*}, x^{*}\right)$. So $d\left(T_{i} x^{*}, x^{*}\right)=0$ since $\lambda<1$, therefore $x^{*} \in T_{i} x^{*}$ by Lemma 1.21$)$.

$$
\text { If } \begin{aligned}
u_{j, n_{k}+1}\left(z, x_{n_{k}+1}\right)=\frac{d\left(z, T_{n_{k}+1} x_{n_{k}+1}\right)}{2} & \begin{aligned}
2 & \left(x_{n_{k}+1}, T_{j} z\right) \\
d\left(T_{i} x^{*}, x^{*}\right) & \leq \frac{\lambda}{2}\left[d\left(z, T_{n_{k}+1} x_{n_{k}+1}\right)+d\left(x_{n_{k}+1}, T_{j} z\right)\right]+d\left(x_{n_{k}+1}, x^{*}\right) \\
& \leq \frac{\lambda}{2}\left[d\left(z, x_{n_{k}}\right)+d\left(x_{n_{k}+1}, x^{*}\right)\right]+d\left(x_{n_{k}+1}, x^{*}\right) .
\end{aligned}
\end{aligned}
$$


Let $k \rightarrow \infty$, then $d\left(T_{i} x^{*}, x^{*}\right) \leq \frac{\lambda}{2} d\left(z, x^{*}\right)$ since $x_{n} \rightarrow x^{*}$, hence similarly, $x^{*} \in T_{i} x^{*}$.

So in any situation, $x^{*} \in T_{i} x^{*}$ for all $i \in \mathbb{N}$, so $x^{*}$ is a common fixed point of $\left\{T_{i}\right\}_{i \in \mathbb{N}}$. If $x^{*}$ and $y^{*}$ are all common fixed points of $\left\{T_{i}\right\}_{i \in \mathbb{N}}$, then we will have

$$
d\left(x^{*}, y^{*}\right) \leq \lambda u_{1,2}\left(x^{*}, y^{*}\right),
$$

where

$$
\begin{aligned}
& u_{1,2}\left(x^{*}, y^{*}\right) \in\left\{\frac{d\left(x^{*}, T_{1} x^{*}\right)+d\left(y^{*}, T_{2} y^{*}\right)}{2}, \frac{d\left(x^{*}, T_{2} y^{*}\right)+d\left(y^{*}, T_{1} x^{*}\right)}{2}\right\} . \\
& \text { If } u_{1,2}\left(x^{*}, y^{*}\right)=\frac{d\left(x^{*}, T_{1} x^{*}\right)+d\left(y^{*}, T_{2} y^{*}\right)}{2} \text {, then } d\left(x^{*}, y^{*}\right)=0 \text {, hence } x^{*}=y^{*} ; \\
& \text { If } u_{1,2}\left(x^{*}, y^{*}\right)=\frac{d\left(x^{*}, T_{2} y^{*}\right)+d\left(y^{*}, T_{1} x^{*}\right)}{2}, \text { then } d\left(x^{*}, y^{*}\right) \leq \frac{\lambda}{2}\left[d\left(x^{*}, T_{2} y^{*}\right)+d\left(y^{*}, T_{1} x^{*}\right)\right]=\lambda d\left(x^{*}, y^{*}\right),
\end{aligned}
$$
hence $d\left(x^{*}, y^{*}\right)=0$ since $\lambda<1$, so $x^{*}=y^{*}$.

Hence in any situation, $x^{*}=y^{*}$. So $x^{*}$ is the unique common fixed points of $\left\{T_{i}\right\}_{i \in \mathbb{N}}$.

If the mappings in Theorem 2.1 are all single-valued, then Theorem 2.1 becomes the next form.

Theorem 2.2. Let $K$ be a nonempty closed subset of a complete metrically convex space $(X, d)$ with $\operatorname{int} K \neq \varnothing,\left\{T_{i}: X \rightarrow X\right\}_{i \in \mathbb{N}}$ a countable family of surjective single-valued mappings such that for any $i, j \in \mathbb{N}$ with $i<j$, any $x, y \in X$,

$$
d(x, y) \leq \lambda u_{i, j}(x, y)
$$

where $u_{i, j}(x, y) \in\left\{\frac{d\left(x, T_{i} x\right)+d\left(y, T_{j} y\right)}{2}, \frac{d\left(x, T_{j} y\right)+d\left(y, T_{i} x\right)}{2}\right\}$ and $\lambda \in\left(0, \frac{-3+\sqrt{17}}{2}\right)$ is a constant number.

Furthermore, if $T_{i}\left(K^{C}\right) \bigcap \partial K=\varnothing$ for all $i \in \mathbb{N}$, and for each $i \in \mathbb{N}$ and $p \in K$, there exists $j \in \mathbb{N}$ such that $p=T_{j} T_{i} p$, then $\left\{T_{i}\right\}_{i \in \mathbb{N}}$ has a unique common fixed point in $K$.

From Theorem 2.2, we can obtain the following more generalized common fixed point theorem.

Theorem 2.3. Let $K$ be a nonempty closed subset of a complete metrically convex space $(X, d)$ with $\operatorname{int} K \neq \varnothing,\left\{T_{i, j}: X \rightarrow X\right\}_{\dot{y}_{j \in \mathbb{N}}}$ a family of subjective single-valued mappings, $\left\{m_{i, j}\right\}_{i, j \in \mathbb{N}}$ a family of positive integral numbers such that for any $i_{1}, i_{2}, j \in \mathbb{N}, \quad x, y \in X$,

$$
d(x, y) \leq \lambda u_{i_{1}, i_{2}, j}(x, y),
$$

where $u_{i_{1}, i_{2}, j}(x, y) \in\left\{\frac{d\left(x, T_{i_{1}, j}^{m_{i_{1}, j}} x\right)+d\left(y, T_{i_{2}, j}^{m_{i, j}} y\right)}{2}, \frac{d\left(x, T_{i_{2}, j}^{m_{2}, j} y\right)+d\left(y, T_{i_{1}, j}^{m_{i_{1}, j}} x\right)}{2}\right\}$ and $\lambda \in\left(0, \frac{-3+\sqrt{17}}{2}\right)$ is a constant number. Furthermore, if 1) $T_{i, j}^{m_{i, j}}\left(K^{C}\right) \cap \partial K=\varnothing$ for all $\left.i \in \mathbb{N}, 2\right)$ for each $i_{1}, j \in \mathbb{N}$ and $p \in X$, there exists $i_{2} \in \mathbb{N}$ such that $p=T_{i_{2}, j}^{m_{i_{2}, j}} T_{i_{1}, j}^{m_{i_{1}, j}} p$, 3) for each $i_{1}, i_{2}, \mu, v \in \mathbb{N}$ with $\mu \neq v, T_{i_{1}, \mu} T_{i_{2}, v}=T_{i_{2}, v} T_{i_{1}, \mu}$. Then $\left\{T_{i, j}\right\}_{i, j \in \mathbb{N}}$ has a unique common fixed point in $K$.

Proof Fix $j \in \mathbb{N}$, and let $S_{i, j}=T_{i, j}^{m_{i, j}}$, then $\left\{S_{i, j}\right\}_{i \in \mathbb{N}}$ satisfies all of the conditions of Theorem 2.2, hence $\left\{S_{i, j}\right\}_{i \in \mathbb{N}}$ has an unique common fixed point $p_{j}$ in ${ }_{i \in \mathbb{N}}$. Now, we will prove that $p_{j}$ is also unique common fixed point of $\left\{T_{i, j}\right\}_{i \in \mathbb{N}}$. In fact, for any fixed $i \in \mathbb{N}$,

$S_{i, j}\left(T_{i, j}\left(p_{j}\right)\right)=T_{i, j}^{m_{i, j}}\left(T_{i, j}\left(p_{j}\right)\right)=T_{i, j}\left(T_{i, j}^{m_{i, j}}\left(p_{j}\right)\right)=T_{i, j}\left(S_{i, j}\left(p_{j}\right)\right)=T_{i, j}\left(p_{j}\right)$. This means that $T_{i, j}\left(p_{j}\right)$ is a fixed point of $S_{i, j}$. For any $k \in \mathbb{N}$ with $k \neq i$, there exists $k^{\prime} \in \mathbb{N}$ such that $S_{k^{\prime}, j} S_{k, j}\left(T_{i, j}\left(p_{j}\right)\right)=T_{i, j}\left(p_{j}\right)$ by 2), and by (3) we have that

$$
d\left(T_{i, j}\left(p_{j}\right), S_{k, j}\left(T_{i, j}\left(p_{j}\right)\right)\right) \leq \lambda u_{k, k^{\prime} j}\left(T_{i, j}\left(p_{j}\right), S_{k, j}\left(T_{i, j}\left(p_{j}\right)\right)\right),
$$

where 


$$
\begin{aligned}
& u_{k, k^{\prime} j}\left(T_{i, j}\left(p_{j}\right), S_{k, j}\left(T_{i, j}\left(p_{j}\right)\right)\right) \\
& \in\left\{\frac{d\left(T_{i, j}\left(p_{j}\right), S_{k, j}\left(T_{i, j}\left(p_{j}\right)\right)+d\left(S_{k, j}\left(T_{i, j}\left(p_{j}\right)\right)\right), S_{k^{\prime}, j}\left(S_{k, j}\left(T_{i, j}\left(p_{j}\right)\right)\right)\right)}{2},\right. \\
& \left.\frac{d\left(T_{i, j}\left(p_{j}\right), S_{k^{\prime}, j}\left(S_{k, j}\left(T_{i, j}\left(p_{j}\right)\right)\right)\right)+d\left(S_{k, j}\left(T_{i, j}\left(p_{j}\right)\right), S_{k, j}\left(T_{i, j}\left(p_{j}\right)\right)\right)}{2}\right\} \\
& =\left\{0, d\left(T_{i, j}\left(p_{j}\right), S_{k, j}\left(T_{i, j}\left(p_{j}\right)\right)\right)\right\} .
\end{aligned}
$$

If $u_{k, k^{\prime}}\left(T_{i, j}\left(p_{j}\right), S_{k, j}\left(T_{i, j}\left(p_{j}\right)\right)\right)=0$, then $d\left(T_{i, j}\left(p_{j}\right), S_{k, j}\left(T_{i, j}\left(p_{j}\right)\right)\right)=0$, hence $T_{i, j}\left(p_{j}\right)=S_{k, j}\left(T_{i, j}\left(p_{j}\right)\right)$;

If $u_{k, k^{\prime} j}\left(T_{i, j}\left(p_{j}\right), S_{k, j}\left(T_{i, j}\left(p_{j}\right)\right)\right)=d\left(T_{i, j}\left(p_{j}\right), S_{k, j}\left(T_{i, j}\left(p_{j}\right)\right)\right)$, then

$d\left(T_{i, j}\left(p_{j}\right), S_{k, j}\left(T_{i, j}\left(p_{j}\right)\right)\right) \leq \lambda d\left(T_{i, j}\left(p_{j}\right), S_{k, j}\left(T_{i, j}\left(p_{j}\right)\right)\right)$, hence $T_{i, j}\left(p_{j}\right)=S_{k, j}\left(T_{i, j}\left(p_{j}\right)\right)$.

Hence in any situation, we have that $T_{i, j}\left(p_{j}\right)$ is a fixed point of $S_{k, j}$ for each $k$ with $k \neq i$. So $T_{i, j}\left(p_{j}\right)$ is a common fixed point of $\left\{S_{i, j}\right\}_{i \in \mathbb{N}}$. By uniqueness of common fixed points of $\left\{S_{i, j}\right\}_{i \in \mathbb{N}}$, we have $T_{i, j}\left(p_{j}\right)=p_{j}$ for each $i \in \mathbb{N}$. Hence $p_{j}$ is a common fixed point of $\left\{T_{i, j}\right\}_{i \in \mathbb{N}}$.

If $u_{j}$ and $v_{j}$ are all common fixed points of $\left\{T_{i, j}\right\}_{i \in \mathbb{N}}$, then they are also common fixed points of $\left\{S_{i, j}\right\}_{i \in \mathbb{N}}$, hence by the uniqueness of common fixed points of $\left\{S_{i, j}\right\}_{i \in \mathbb{N}}$, we obtain $u_{i}=p_{j}=v_{j}$. This means that for each $j \in \mathbb{N},\left\{T_{i, j}\right\}_{i \in \mathbb{N}}$ has a unique common fixed point $p_{j}$.

Now, we prove $p_{\mu}=p_{v}$ for each $\mu, v \in \mathbb{N}$. In fact, for any $i_{1}, i_{2}, \mu, v \in \mathbb{N}$ with $\mu \neq v$, since $T_{i_{1}, \mu}\left(p_{\mu}\right)=p_{\mu}$ and $T_{i_{2}, v}\left(p_{v}\right)=p_{v}$, so $T_{i_{1}, \mu}\left(T_{i_{2}, v}\left(p_{v}\right)\right)=T_{i_{1}, \mu}\left(p_{v}\right)$, hence

$T_{i_{2}, v}\left(T_{i_{1}, \mu}\left(p_{v}\right)\right)=T_{i_{1}, \mu}\left(T_{i_{2}, v}\left(p_{v}\right)\right)=T_{i_{1}, \mu}\left(p_{v}\right)$ by 3). Therefore, $T_{i_{1}, \mu}\left(p_{v}\right)$ is a fixed point of $T_{i_{2}, v}$ for each $i_{2}$, i.e., $T_{i_{1}, \mu}\left(p_{v}\right)$ is a common fixed point of $\left\{T_{i_{2}, v}\right\}_{i_{2} \in \mathbb{N}}$. But $\left\{T_{i_{2}, v}\right\}_{i_{2} \in \mathbb{N}}$ has a unique common fixe point $p_{v}$, hence $T_{i_{1}, \mu}\left(p_{v}\right)=p_{v}$ for each $i_{1}$, and therefore $p_{v}$ is a common fixed point of $\left\{T_{i_{1}, \mu}\right\}_{i_{1} \in \mathbb{N}}$. But $\left\{T_{i_{1}, \mu}\right\}_{i_{1} \in \mathbb{N}}$ has a unique common fixed point $p_{\mu}$, hence $p_{\mu}=p_{v}$. Let $p^{*}=p_{j}$, then $p^{*}$ is the common fixed point of $\left\{T_{i, j}\right\}_{i, j \in \mathbb{N}}$. The uniqueness of common fixed points of $\left\{T_{i, j}\right\}_{i, j \in \mathbb{N}}$ is obvious.

\section{Funding}

This work was supported by the National Natural Science Foundation of China (No. 11361064).

\section{REFERENCES}

[1] N. A. Assad, “On Fixed Point Theorem of Kannan in Banach Spaces,” Tamkang Journal of Mathematics, Vol. 7, 1976, pp. 91-94.

[2] N. A. Assad, "Fixed point Theorems for Set-Valued Transformations on Compact Sets,” Bolletino della Unione Matematica Italiana, Vol. 7, No. 4, 1973, pp. 1-7.

[3] N. A. Assad and W. A. Kirk, "Fixed Point Theorems for Set-Valued Mappings of Contractive Type,” Pacific Journal of Mathematics, Vol. 43, No. 3, 1972, pp. 553-562. http://dx.doi.org/10.2140/pjm.1972.43.553

[4] X. Zhang, “Common Fixed Point Theorem of Lipschitz Type Mappings on Convex Cone Metric Spaces,” Acta Mathematica Sinica (Chinese Series), Vol. 53, No. 6, 2010, pp. 1139-1148.

[5] M. Abbas, B. E. Rhoades, et al., "Common Fixed Points of Generalized Contractive Multivalued Mappings in Cone Metric Spaces,” Mathematical Communications, Vol. 14, No. 2, 2009, pp. 365-378.

[6] S. L. Singh and B. Ram, "Common Fixed Points of Commuting Mappings in 2-Metric Spaces,” Mathematical Semester Notes, Vol. 10, 1982, pp. 197-207. 
[7] Y. J. Piao and Y. F. Jin, "New Unique Common Fixed Point Results for Four Mappings with $\Phi$-Contractive Type Theorems in 2-Metric Spaces,” Applied Mathematics, Vol. 3, No. 7, 2012, pp. 734-737. http://dx.doi.org/10.4236/am.2012.37108

[8] M. S. Khan, H. K. Pathak and M. D. Khan, “Some Fixed Point Theorems in Metrically Convex Spaces,” Georgian Mathematical Journal, Vol. 7, No. 3, 2000, pp. 523-530.

[9] S. K. Chatterjea, “Fixed Point Theorems,” Comptes rendus de l'Académie des Sciences, Vol. 25, 1972, pp. 727-730.

[10] O. Hadzic, “Common Fixed Point Theorem for a Family of Mappings in Convex Metric Spaces,” Univ. U. Novom Sadu, Zb. Rad. Prirod. Mat. Fak. Ser. Mat., Vol. 20, No. 1, 1990, pp. 89-95.

[11] Y. J. Piao, “Unique Common Fixed Point Theorems for a Family of Non-Self Maps in Metrically Convex Spaces,” Applied Mathematics, Vol. 22, No. 4, 2009, pp. 852-857.

[12] Y. J. piao, "Unique Common Fixed Point Theorems for a Family of Quasi-Contractive Type Maps in Metrically Convex Spaces,” Acta Mathematica Scientia, Vol. 30A, No. 2, 2010, pp. 487-493.

[13] J. R. Wu and H. Y. Liu, “Common Fixed Point Theorems for Sequences of $\Phi$-Type Contraction Set-Valued Mappings," Chinese Quarterly Journal of Mathematics, Vol. 24, No. 4, 2009, pp. 504-510. 